\title{
A Note on the (Faith-Menal) Counter Example
}

\author{
R. H. Sallam \\ Mathematics Department, Faculty of Science, Helwan University, Cairo, Egypt \\ Email: rsallams@hotmail.com
}

Received September 1, 2011; revised October 15, 2011; accepted October 22, 2011

\begin{abstract}
Faith-Menal counter example is an example (unique) of a right John's ring which is not right Artinian. In this paper we show that the ring $\mathrm{T}$ which considered as an example of a right Johns ring in the (Faith-Menal) counter example is also Artinian. The conclusion is that the unique counter example that says a right John's ring can not be right Artinian is false and the right Noetherian ring with the annihilator property $\mathrm{rl}(\mathrm{A})=\mathrm{A}$ may be Artinian.
\end{abstract}

Keywords: John's Ring; Artinian and Noetherian Rings

\section{Introduction}

A ring $\mathrm{R}$ is called right John's ring if it is right Noetherian and every right Ideal $\mathrm{A}$ of $\mathrm{R}$ is a right annihilator i.e. $\mathrm{rl}(\mathrm{A})=\mathrm{A}$ for all right ideals $\mathrm{A}$ of $\mathrm{R}$.

John's ([1], Theorem 1) by using a result of Kurshan ([2], Theorem 3.3), showed that a right Noetherian ring is right Artinian provided that every right ideal is a right annihilator.

Ginn [3] showed that Kurshan result was false. Ginn's example does not provide a counter example to John's theorem. Therefore the validity of John's theorem was doubtful.

Faith-Menal counter example proved that there is an example (unique) of a right John's ring which is not right Artinian.

Here in this paper we prove the false of the FaithMenal counter example by proving that the considered non-Artinian right john's ring is in fact right Artinian. So the John's theorem may be true see [1].

All rings considered in this paper are associative rings with identity.

We recall the Faith-Menal counter example in Section 1 and we prove that it is false in Section 2.

\section{Section 1: The Counter Example}

Example 8.16 (Faith-Menal) [4]. Let D be any countable, extentially closed division ring over a field $\mathrm{F}$, and let $\mathrm{R}=$ $\mathrm{D} \otimes_{\mathrm{F}} \mathrm{F}(\mathrm{x})$. Then $\mathrm{T}(\mathrm{R}, \mathrm{D})$ is a non-Artinian right John's ring.

\section{Proof}

Cohn shows that $\mathrm{R}$ is simple, principle right ideal domain that is right $\mathrm{V}$ ring (Theorems 8.4.5 \& 5.5.5 [4]) and $\mathrm{D}$ is an R-R bimodule such that $\mathrm{D}_{\mathrm{R}}$ is the unique simple right $\mathrm{R}$ module. Hence T(R, D) is a right John's ring by Theorem 8.15 (in this book). But $\mathrm{T}(\mathrm{R}, \mathrm{D})$ is not Artinian because if it were then $\mathrm{R}$ would also right Artinian and hence a field which is a contradiction.

Here

$$
\mathrm{T}=\mathrm{R} \oplus \mathrm{D} \text { and } \mathrm{T} /\langle 0, \mathrm{~d}\rangle \cong \mathrm{R}
$$

For more information about this example see [4].

\section{Section 2: A Note on the Counter Example}

\section{Theorem}

The right John's ring $T(R, D)$ defined in the counter example is Artinian.

\section{Proof}

Recall the following (Exercises 10.7 [5]):

Let $\varphi: \mathrm{D} \rightarrow \mathrm{R}$ be a ring homomorphism and let $\mathrm{M}$ be a right R-module (or left R-module) then

1) Via $\varphi M$ is right $D$-module;

2) If $M_{D}$ is Artinian or Noetherian then so is $M_{R}$;

3) If $\mathrm{R}$ is finite dimensional algebra (via $\varphi$ ) over a field $\mathrm{D}$ then the following is equivalent:

a) $M_{R}$ is Artinian and Noetherian

b) $M_{R}$ is finitely generated

c) $\mathrm{M}_{\mathrm{D}}$ is finite dimension

1) Consider the ring homomorphism $\varphi: D \rightarrow R$ defined by $\varphi(\mathrm{d})=\mathrm{d} \otimes 1_{\mathrm{f}}$. Every R-module homomorphism is a $D$-homomorphism via $\varphi$. Since D is a division ring so it will be semisimple ring and hence every right $\mathrm{D}$-module is semisimple (Corollary 8.2.2 [3]).

2) If the ring $T=R \oplus D$ is John's ring (as in the counter example above) then it is Noetherian and hence $\mathrm{R}$ and $\mathrm{D}$ are Noetherian. As D is right Noetherian ring then every finitely generated right $\mathrm{D}$ module is Noetherian (6.1.3 [6]).

3) Every finitely generated right $\mathrm{D}$-module $\mathrm{M}$ is semisimple so it is right Artinian. 
4) Since every finitely generated right $D$-module $M$ is Artinian and Noetherian then M is Artinian and Noetherian as an R-module.

5) Now since $R$ is simple principle ideal domain then $\mathrm{R}$ is a finite dimensional $\mathrm{k}$-algebra where $\mathrm{k}$ is a subring of the center of $R$ with identity $1_{R}$ and hence $R$ is a finite dimensional algebra over the field $\mathrm{Z}$ (D) the center of $\mathrm{D}$.

Applying the above equivalence we get that every finitely generated right R-module is Artinian and hence $\mathrm{R}$ is right Artinian and this imply that $\mathrm{T}$ is also Artinian.

\section{Conclusion}

The conclusion is that the unique example that says a right John's ring can not be right Artinian is false.

\section{REFERENCES}

[1] B. Johns, "Annihilator Conditions in Noetherian Rings,"
Journal of Algebra, Vol. 49, No. 1, 1977, pp. 222-224. doi:10.1016/0021-8693(77)90282-4

[2] R. P. Kurshan, "Rings Whose Cyclic Modules Have Finitely Generated Socle," Journal of Algebra, Vol. 15, No. 3, 1970, pp. 376-386. doi:10.1016/0021-8693(70)90066-9

[3] S. M. Ginn, "A Counter Example to a Theorem of KurshAn," Journal of Algebra, Vol. 40, No. 1, 1976, pp. 105-106. doi:10.1016/0021-8693(76)90090-9

[4] W. K. Nicholson and M. F. Yousif, "Quasi-Frobenius Rings," Series Cambridge Tracts in Mathematics, No. $158,2003$.

[5] F. W. Anderson and K. R. Fuller, "Rings and Categories of Module," Springer Verlag, New York, 1991.

[6] F. Kasch, "Modules and Rings, London Mathematical Society Monographs," Vol. 17, Academic Press, New York, 1982 